kilowatts have been developed. But the figure is probably a gross under-estimate as in the case of the U.S.S.R. The first problem before the Government of India should be, therefore, to carry out an adequate survey of the hydro-electric resources of India, and plan for the development of ample power for industries.

Civilized life in India has, from time immemorial, grown in river-valleys, which have been used for navigation and irrigation. During the British regime, development has been one-sided, namely, for irrigation only, and navigation has been allowed to fall into decay. But both Soviet Russia and the United States of America have, within the last twenty-five years, set a new ideal for river development. We can take, as a typical illustration, the development of the Tennessee River, which before 1933 was a matter of great concern to the Federal Government on account of the destructive floods, soil erosion, and progressive pauperization of dwellers of the valley. But it is now well known, thanks to the initiative of American statesmen, that this river has been completely transformed by the construction of twenty-one dams over the main river and tributaries. These dams serve the multiple purpose of flood-prevention, navigation and power development, and soil erosion has been prevented by auxiliary measures. The hitherto untamed river, instead of being allowed to dissipate its energy on destructive work, is now harnessed and yields 12,000 million units of electrical energy, which is used for great productive works which have sprung up in the valley (metallurgical, chemical and fertilizer industries).

The multi-purpose development of a river valley, the benefits of which have been so strikingly demonstrated by the Tennessee Valley Authority, is applicable to at least a score of river valleys in India. I have made a particular study of the Damodar Valley, an area of 9,000 square miles, which forms the country to the west of the great city of Calcutta. The lower part of this valley, forming the suburban area of Calcutta, was, a hundred years ago, one of the healthiest regions of the world. But after the construction of railways from 1850, and great roads to connect Calcutta with upper India, embankments were reinforced and constructed on the left bank of the river in order to protect these highways from the destructive river floods. These embankments have by no means removed the danger to the highways; for in 1943 there was only a moderate flood which breached the line at numerous points, and seriously interfered with traffic to Calcutta at a very critical period of the history of the country. They have, in addition, turned the country into a malarial swamp. Even the safety of Calcutta is endangered.

I have shown by a preliminary study that this valley can be subjected to the same treatment as the Tennessee, and can be developed for energy generation (to the extent of 2,000 million $\mathrm{kW}$. a year), navigation, and flood prevention. The lower valley can be transformed by auxiliary methods of irrigation into a fine suburban area, where the teeming population of Calcutta can expand.

But the Damodar Valley is not unique. The Sone, the rivers of the Deccan (the Kaveri has been developed to some extent), the Mahanadi, the Punjab rivers, and many others too numerous to mention, can be developed according to the T.V.A. methods for the benefit of India's millions.

This is only a cursory survey of the problems of India and of the way in which we have to look at it.
It is regrettable that the Indian leaders have so far paid attention only to the question of political freedom. It is natural that everyone of us should want our country to attain full nationhood and the people have full sovereignty, but the problem of living for millions of Indians cannot be postponed; in fact, we believe that the only way to achieve unity of thought and purpose in the political field, which is now wanting, is first to look at the problem of living for India's millions. To solve this problem successfully there must be a national purpose behind all planning, and I do not see how any planning can be given effect without a National Government, or unless we have a Government which has popular support and is composed of leaders in whom the people have confidence.

\section{ASTRONOMICAL AND GEOPHYSICAL PERIODICITIES}

A GEOPHYSICAL Discussion on "Astronomical A and Geophysical Periodicities" was held in the rooms of the Royal Astronomical Society on December 8, 1944 ; the chair was taken by Prof. L. M. Milne-Thomson, and in the absence of Dr. Harold Jeffreys through indisposition, the discussion was opened by Dr. H. R. Hulme.

Dr. Hulme's interest in this problem originally arose in connexion with the reality of the supposed periodical variations in the solar constant. Any time-series of observations can be analysed by the routine method of harmonic analysis, and amplitudes will be obtained which will not in general be zero, even when there is not the slightest reason for suspecting the presence of a periodic variation; such an analysis has, in fact, often been carried out with geophysical data. The first attempt to find a criterion for the reality of a periodic term was made in 1906 by Sir Arthur Schuster, who found that if $n$ independent observations were analysed into their Fourier components the average amplitude $\rho$ of a Fourier coefficient is $2 \sigma / n^{1 / 2}$, where $\sigma$ is the standard deviation of the $n$ observations ; if, then, the observed amplitude is $p_{0}$, the chance that such a component could arise purely as a result of random fluctuations is $e^{-k}$, where $k=\rho_{0}^{2} / \rho^{2}$. Thus, if the amplitude found is more than twice $p$, there is some ground for suspecting that the periodicity may be real.

The Schuster criterion, however, takes no account of internal correlations among the data. Thus a naive view of the problem may give misleading results ; if, for example, the observations are subject to disturbances, each of which affects a number of consecutive observations, the effective number of independent observations may be far less than $n$. Moreover, the criterion is of limited usefulness, since an investigator who thought that the values of a series of observations were all independent of one another would not be likely to make a harmonic analysis of the results. A more searching method of analysing a time-series was given in 1927 by $\mathrm{Mr}$. G. Udny Yule. He considered two types of periodicities : the periodic phenomena where each disturbance affects only the momentary value of the quantity observed; and periodic phenomena in which each disturbance affects all the subsequent values, as, for example, a change of phase. The latter type would be discussed by Mr. M. G. Kendall. Dr. Hulme pointed out that an outstanding problem is to find 
how to test whether a given oscillation belongs to one or other of these types.

Mr. H. W. Newton stated that more than two hundred years elapsed between the discovery of sunspots and the discovery of their eleven-year periodicity; the success of Schwabe in discovering the eleven-year cycle in 1610 might be attributed to his systematic work and to the fact that he continued his observations over three or four solar cycles. A graph of the frequency of naked-eye sunspots indicates that an observer with a piece of smoked glass making systematic observations like Schwabe could have discovered the eleven-year cycle; it is possible, however, that observers in the seventeenth century may have been frustrated by a 'submergence' of the sunspot cycle. The observed reversal of the polarity of the magnetic field in sunspots suggests that the fundamental period is twenty-two years; this, however, is not obvious from the diagram of many sunspot cycles as measured at Greenwich, though Turner got a difference from spot area data, 18421910, nor does it show up in Maunder's famous diagram (the 'butterfly' diagram) of the cyclical change of latitude of sunspots.

The Zurich 'sunspot numbers' from 1750 until 1911 were subjected to a harmonic analysis by Kimura. As in the customary method of predicting tidal heights, Kimura computed the form of the extrapolated curve after 1911; this prediction was not realized, for the time and amplitude of the next following maximum observed in 1917 were in marked disagreement with Kimura's values. Other analyses had been made by Newcomb, Schuster, Yule, Waldmeier and others; the average length of the cycle may be accepted as 11-13 years. Mr. Newton emphasized the necessity of using a long series of data in any attempt to establish a periodicity or a correlation; for example, the annual excess of turns of a weather vane at Greenwich, direct over retrograde, had been compared with the sunspot cycle, and it was found, rather surprisingly, that a general parallelism existed for some years. This correspondence, however, did not persist, and the parallelism appeared to be quite fortuitous. In conclusion, $\mathrm{Mr}$. Newton showed a diagram of the comparison over thirty years of the monthly sunspot areas and the diurnal inequality ranges in magnitude of the terrestrial magnetic elements $D, H$ and $V$. The correspondence is very striking, and extends to the details of the curves; later observations confirm the closeness of the correlation, and give values as high as 0.95 for the coefficient of correlation for individual eleven-year cycles.

Mr. M. G. Kendall, in his contribution, explained that although he knew little about astronomy, he believes that many of the oscillatory movements in time-series which are found in geophysical and meteorological data possess similar features to those in economic series in which he is primarily interested. In this connexion he, wished to direct attention to the large amount of work which is being done on time-series in quite unrelated fields, the necessity for an interchange of information between workers in different branches of science, and some co-ordination of effort.

The classical method of analysing oscillatory movements is to exhibit the series as a sum of harmonic terms. If, as usually happens, observation differs from mathematical representation, the differences, however large, are regarded as errors of observation in the sense that their effect is instantaneous and does not endure in the future motion of the system. For most practical series, this does not appear to be a plausible hypothesis, because disturbances of the motion, though possibly random in the sense that their occurrence is according to the laws of chance, permanently affect the future motion of the system and are incorporated into it.

The statistical problem is to find a mathematical representation of such a system. The nearest approach to a solution so far advanced was that given in 1927 by Mr. Udny Yule in his paper on sunspots. Yule was led to consider a type of series, which has been called 'auto-regressive', in which the value at any point is partly a function of those at previous points, and partly a disturbance function. One simple form of this type of series can be written

$$
u_{t+2}+a u_{t+1}+b u_{t}-\varepsilon_{t+2}=0,
$$

where $\varepsilon_{t+2}$ is the disturbance, which can in particular cases be random. Mr. Kendall has recently constructed a number of artificial series of this type and analysed them by the Schuster periodogram method. The results are very striking. Not only does the periodogram fail to reveal the true nature of the series, but also it suggests quite a large number of periods where none exist. He has come to the conclusion that for series of this type periodogram analysis is not worth the labour of undertaking.

Mr. Kendall has also considered the practice of counting peaks in a series as providing an estimate of 'period'. For autoregressive series of the type mentioned, it appears that this is an extremely insensitive method inasmuch as for the majority of values likely to be encountered in practice it will give a value somewhere between 4 or 6 units, whatever the nature of the series. His experimental series and a number of practical economic series do in fact give such values, and the appearance of 'periods' of this kind throws very little light on the true nature of the generating process.

Reference was also made by Mr. Kendall to the technique introduced by Mr. Yule of computing the serial correlations of the series. This appeared to give much more reliable results, but is not without its difficulties. In conclusion, he said that in his. view a great deal of the work which has been done on the analysis of oscillatory movements will have to be reconsidered; he is convinced that 90 per cent of the 'periods' which have been claimed by different writers were spurious.

Miss N. Carruthers discussed periodicities in weather phenomena. She said that there are some true periodicities, such as, for example, the annual and diurnal variation of most meteorological elements, but it is doubtful whether they are truly represented by combinations of sine curves. In addition to these, spasmodic oscillations are found in most series of meteorological data. Some may be purely random fluctuations, but others have a tendency to reappear at intervals with the same wave-length, although not, in general, with the same phase. The latter most often persist for three or four wave-lengths only, after which they either end abruptly or change phase unexpectedly. Examining meteorological waves (the irregular kind can scarcely be termed periodicities) with Dr. C. E. P. Brooks, it had been found that determination by harmonic analysis is unreliable, and a year ago they devised the 'periodoscope' which Miss Carruthers described before the Royal Meteorological Society last June. In this form of analysis, by a simple combination of the terms in a series 
containing periodicities, a new series is formed in which these were retained with period and phase unaltered. For periods lying within a pre-assigned range, however, amplitudes are magnified, so that, when the new series is plotted, the corresponding periodicities can be readily identified by eye with the aid of key curves drawn on tracing linen. Examples of waves found by this means in London temperature were contrasted with the more regular sunspot cycle (9-13 years) treated in the same way.

Most waves in meteorological phenomena appear to be natural oscillations of the earth's atmosphere. Haurwitz, Lettau and Defant deduced theoretical periods of 6-57 days, all of which have been found in pressure and some also in rainfall. These, however, are not set up without some external stimulus, just as a violin string does not emit its characteristic note of its own accord. The fragmentary nature of these waves can, likewise, be explained by analogy with a violin string ; for if the string receives impulses at intervals incommensurate with its period of vibration, the vibrations break up and change phase abruptly with each fresh impulse. The analogous impulses received by the atmosphere appear to be connected with variations of solar radiation. That these occur at intervals which are not natural periods of atmospheric vibration is indicated by a comparison of prevailing periods in the solar constant and in European pressure :

$$
\begin{array}{lll}
\text { Solar Constant } & . & 75,51,37,25,19 \\
\text { Pressure days }
\end{array}
$$

A further illustration is afforded by the 3-year wave in the pressure at Darwin, which has been found by C. Braak to break up and change phase about the time of sunspot maximum; this is probably the same as the waves found earlier in India and the Argentine by Sir Norman Lockyer and identified as $3 \frac{1}{2}$-year periodicities.

Other periods indirectly induced by solar radiation are connected with interchange of air between continents and oceans and outbreaks of cold air from the polar regions. The moon induces a semidiurnal atmospheric tide which is a true periodicity, but it is too small to have any appreciable effect on the weather.

Mr. P. M. Ryves summarized the general characteristies of stars the intrinsic luminosity of which is variable; these constitute only a small percentage of the stars observed, but they cover the whole range of spectral classes from $O$ to $M$ and $N$. Periods range from $1 \frac{1}{2}$ hours to 20 years or more, but periodicities of more than two years generally correspond to small amplitude of variation, and are often irregular or uncertain, and frequently complicated with superimposed shorter periods. There is a very definite correlation between length of period and spectral class, the shorter periods corresponding to the hotter early-type stars and long periods to late types. Though variables can be found with periods anywhere between the limits mentioned, a frequency curve shows three prominent peaks, one corresponding to the RR Lyrids with mean period of about half a day, the second to the Cepheids (really a double peak with a minimum at about nine days), and the third, at a little short of 300 days, to the Mira-type and other long-period variables.

Dealing particularly with the long-period variables, Mr. Ryves mentioned that typical Mira-type stars have a mean amplitude of about five magnitudes, with spectral class $M 3$ to $M 8$ (90 per cent), or $N$
(5 per cent) or $S$ (5 per cent), all with emission spectrum, and periods for the most part between 150 and 550 days. The magnitude at maximum varies in individual cycles to the extent of one magnitude or more, and the magnitude at minimum also varies, but generally to a smaller extent. The period is frequently irregular in the sense that any given phase may be unpunctual to the extent of 5-10 per cent, but the mean period is fairly constant. Apart from these oscillations about the mean, there are sometimes permanent changes: $(a)$ a sudden change in the length of the period (this has happened more than once in some stars); (b) a discontinuity, or shift in the phase, without change of period. Mira-type stars show a small progression of spectral class with increasing length of period, and also, but with many exceptions, a progression from symmetrical to asymmetrical light curves, and increase in amplitude. The shape of the curve may vary a good deal from cycle to cycle, and there is sometimes a pause or hump as in the sunspot curve; this happens more frequently on the ascending than on the descending branch. In some cases the hump is a more or less permanent feature, and shows up on the mean curve. The variables have large space-motions, and are probably revolving about the centre of the galaxy in highly elliptical orbits.

Parallel with the Mira-type variables is another group of stars comprising the red semi-regular variables and the so-called red irregular variables. The fundamental difference is that these have no emission spectrum, or at most a very faint one, and that the amplitude is much smaller, usually one or two magnitudes. The variation is also less regular, both as regards punctuality and the shape of the lightcurve. Double maxima, or a tendency for deep and shallow minima to alternate, are common, and a long-term variation in the median magnitude sometimes appears. With irregular variables there may be several maxima at fairly regular intervals, followed by a period of disturbance, after which a new series of maxima appears, quite out of phase with the former series.

Dr. R. Stoneley recalled a paper by Dr. H. Jeffreys that is extremely relevant to the present discussion. Many harmonic analyses had been made of the occurrence of earthquake shocks, notably by Prof. H. H. Turner and.Dr. C. Davison. The latter had applied the Schuster criterion to his results, and prima facie had found good evidence for the genuineness of a number of the periodicities. Dr. Jeffreys found that the existence of internal correlations among the data would increase the random amplitudes expected in a Fourier analysis, and would therefore vitiate the direct application of the Schuster criterion. The phenomenon of aftershocks following a large earthquake does imply that all the shocks. recorded in a catalogue cannot be treated as random occurrences. In the aftershocks of the Tango earthquake of March 7, 1927, the falling-off of the frequencies of the aftershocks is consistent with a regular law of chance that depends only on the time-intervals elapsing since the main shock and a second strong shock on April 1, 1927, but otherwise the aftershocks appear to be mutually independent. The amplitudes obtained for periodicities superposed on this variation are not such as would give any support for the realities of these periodicities.

As was stated at the beginning of this article, Dr. Jeffreys was not present at the meeting, but was asked to comment on the above report. He emphas- 
izes that the Schuster criterion is the particular case of Pearson's $\chi^{2}$ test when the number of degrees of freedom is 2. The analysis of the variation of latitude observations shows the same kind of serial correlation as Yule has considered, with the additional complication that the disturbances do not appear to be derived from the normal law. Fortunately, it was possible to find a long interval when there seems to have been little disturbance. In this problem there is an observational error special to each datum and also a real fluctuation the successive values of which are correlated; the method of maximum likelihood can deal easily with either by itself but becomes prohibitively complicated when the two are superposed.

\section{H/EMOGLOBIN IN THE ROOT NODULES OF LEGUMINOUS PLANTS}

\author{
By Prof. D. KEILIN, F.R.S. \\ AND \\ DR. Y. L. WANG \\ Molteno Institute, University of Cambridge
}

$\mathrm{T}$ HE red pigment in the root nodules of a leguminous plant (Vicia Faba) was investigatéd for the first time by Pietz ${ }^{1}$. He believed it to be identical with the red intermediate product which appears during the oxidation of tyrosine or of dihydroxyphenylalanine ('dopa') catalysed by tyrosinase. According to Pietz, the red pigment, by undergoing a reversible reduction, has the function of poising the oxido-reduction potential of the nodule at a level favourable for the proliferation of symbiotic bacteria inhabiting the nodule.

Pietz' conclusion, however, was based not upon the direct study of the pigment, but upon certain considerations derived from the study of oxido-reduction potentials of nutrient media in relation to growth of symbiotic micro-organisms, and the fact that the addition of 'dopa' to the medium favours the growth of these bacteria.

A year later the pigment in the root nodules of a great variety of leguminous plants was reinvestigated by $\mathrm{Kubo}^{2}$ both in situ and in preparations obtained by fractionation of an extract of nodules with ammonium sulphate. He examined the pigment spectroscopically and determined the position of its absorption bands under different conditions. Thus when aerated, the pigment shows two absorption bands, at $575 \mathrm{~m} \mu$ and $540 \mathrm{~m} \mu$, which, on reduction with sodium hyposulphite, are replaced by one band at $555 \mathrm{mu}$. In the presence of carbon monoxide the bands are shifted to $570 \mathrm{~m} \mu$ and $535 \mathrm{~m} \mu$, and on treating the pigment with potassium ferricyanide a compound is obtained with three absorption bands, at $625 \mathrm{~m} \mu$, $563 \mathrm{~m} \mu$ and $530 \mathrm{~m} \mu$. This latter compound was found by Kubo to react with cyanide, fluoride and peroxide. Finally he obtained from this pigment crystals of hæmin indistinguishable from protohæmin. As the result of these observations, Kubo concluded that the red pigment of the nodules is a hæmoprotein compound analogous to hæmoglobin and acting as a store as well as a carrier of oxygen.

More recently, the nature of this pigment was reinvestigated by Burris and $\mathrm{Haas}^{3}$ on the material obtained from root nodules of cow-peas (Vigna sinensis) fractionated with ammonium sulphate. The light absorption of the pigment thus obtained showed a strong $\gamma$-band at $405 \mathrm{~m} \mu$ characteristic of hæmatin compounds and in the visible region one band only at $530 \mathrm{~m} \mu$ instead of the two bands described by Kubo. They also found that after "deoxygenation of the solution the spectrum of the oxidized form remains unchanged, indicating true oxidation and not merely oxygenation of the red pigment". On reduction with sodium hyposulphite the $\gamma$-band moved to $430 \mathrm{~m} \mu$, and the band in the visible region was replaced by one at $560 \mathrm{~m} \mu$. Burris and Haas agree with Kubo that the pigment is a hæmin compound, but, contrary to his views, they think that its "behaviour towards oxygen indicates that it is not a hæmoglobin-like substance".

The results of the work summarized above leave no doubt that the red pigment in the root nodules of leguminous plants is a hæmatin compound and not a quinone as was postulated by Pietz. The object of the present investigation was to determine whether the pigment is an oxygen carrier of the hæmoglobin type which combines reversibly with molecular oxygen, or whether it is an oxidation catalyst which, like the components of cytochrome, undergoes a reversible oxido-reduction accompanied by valency changes of the hæmatin iron.

Although we have examined the nodules of a number of leguminous plants, the material we have used for this study was mainly the root nodules of the soya bean. The pigment was studied either directly in nodules compressed between two slides or as extracts of nodules purified by fractional precipitation with ammonium sulphate.

The position of the absorption bands of this pigment and of its derivatives was determined with the microspectroscope, and the absorption spectra were determined with the Hilger-Nutting spectrophotometer. This was done only for the visible region of the spectrum, since hæmatin derivatives are more readily differentiated by the absorption bands in this region than by their $\gamma$-bands.

One of the main difficulties in the extraction of this pigment from nodules, which have to be crushed for this purpose, is the more or less rapid darkening of the pulp and of the extract due to oxidation of a phenolic substance catalysed by the phenol oxidase. The quinone formed during this reaction oxidizes the iron of the pigment and partly denatures its protein, whereas the melanine which is ultimately formed adsorbs the pigment and masks its colour and its absorption spectrum. However, one can overcome these difficulties in several ways. Thus, by crushing the nodules in saturated ammonium sulphate and washing the pulp in the same solution, the phenoloxidase can be precipitated before it has time to act upon the substrate, which can be afterwards removed. At the same time we have added an excess of sodium azide which poisons phenolase and a small amount of sodium hyposulphite which also prevents the oxidation of the substrate and keeps the iron of the pigment in the reduced state. The reduced state can be more efficiently stabilized by saturating the solution during different manipulations with carbon monoxide. Under these conditions the extracts can easily be fractionated with ammonium sulphate, and the fraction between 65 per cent and 84 per cent saturation, which contains most of the red pigment, is collected. 